\title{
Size-Dependent Spin Structures in Iron Nanoparticles
}

\author{
A. Fraile Rodríguez, ${ }^{1, *}$ A. Kleibert, ${ }^{1}$ J. Bansmann, ${ }^{2}$ A. Voitkans, ${ }^{3}$ L. J. Heyderman, ${ }^{1}$ and F. Nolting ${ }^{1}$ \\ ${ }^{1}$ Paul Scherrer Institut, Villigen PSI, CH-5232 Switzerland \\ ${ }^{2}$ Institute of Surface Chemistry and Catalysis, University of Ulm, D-89069 Ulm, Germany \\ ${ }^{3}$ Institute of Physics, University of Rostock, D-18051 Rostock, Germany
}

(Received 7 December 2009; published 24 March 2010)

\begin{abstract}
Using photoemission electron microscopy, we study the magnetization orientation in single 5-25 $\mathrm{nm}$ iron particles coupled to a ferromagnetic cobalt support. We find a noncollinear alignment between the particle and substrate magnetization above a particle size of $\sim 6 \mathrm{~nm}$ and a parallel alignment for smaller sizes. Numerical calculations reveal a transition from an exchange-dominated to an anisotropy-dominated regime on increasing the particle height: the smaller particles are in a single-domain collinear state while larger particles exhibit a spin-spiral magnetic structure determined by the magnetic anisotropy energy.
\end{abstract}

Magnetic nanoparticles exhibit a variety of unusual phenomena when compared to the bulk materials, particularly when the dimensions involved are comparable to critical magnetic length scales such as the exchange length [1]. Below a critical size, the particles are in a singledomain state, and for iron (Fe) nanoparticles, micromagnetic calculations have shown that this single-domain limit occurs at a particle size of about $15 \mathrm{~nm}$ when assuming the bulk values for the magnetic anisotropy energy (MAE) and the exchange interaction [2]. However, larger experimental values for the single-domain limit have been observed [3], and domain walls form in much smaller structures during magnetization reversal [4]. It is therefore not trivial to build a consistent picture of the single-domain limit and of the particle size dependence of the spin arrangement close to the single-domain to multidomain transition.

In addition to this fundamental interest, a clear understanding of spin configurations at the nanoscale is required for improving the performance of materials and devices. For such applications, it is often the effects at the interface between two materials which are critical to their functionality. For example, the energy product in exchange-spring nanocomposites [5] can be enhanced by modifying the hard-soft magnetic interface. On bringing magnetic clusters or nanoparticles into contact with a magnetic substrate, a nanoscale interface is created, providing a means to enhance their magnetic stability against thermal fluctuations [6] and to tailor the spin orientation of nanoparticles, which is defined by the properties of the substrate [7-9]. The magnetic coupling between small structures and a substrate is qualitatively well understood, with the exchange interaction at the interface, albeit short range, being usually strong $(\sim 1 \mathrm{eV}$ per atom) relative to other energy terms such as the MAE (around 10-100 $\mu \mathrm{eV}$ per atom). However, since to a first approximation the MAE scales with the particle size, it is anticipated that above a certain threshold the MAE of the particle may become dominant over the exchange energy and ultimately result in an independent orientation of the particle magnetization with respect to the substrate. A detailed picture of such a sizedependent transition is lacking until now because most experimental studies have been limited to measurements encompassing large ensembles of particles. Therefore, single-particle properties were masked by distributions of particle size, anisotropies, and dissimilar local interactions with the underlying substrate [7-9].

In this work we show that above a critical size of $\sim 6 \mathrm{~nm}$, the competition of exchange interaction and anisotropy energy can lead to the formation of noncollinear spin structures in Fe nanoparticles coupled to a ferromagnetic cobalt (Co) substrate, while the magnetization of the smaller particles is aligned collinearly with that of the substrate. This transition can only be captured by quantitatively determining the magnetization orientation of individual nanoparticles. Numerical calculations indicate a sharp transition from an exchange-dominated to an anisotropy-dominated regime: the smaller particles are in a single-domain state with magnetization collinearly aligned to that of the substrate, while larger particles exhibit a static spin spiral analogous to an exchange spring.

The nanoparticles studied here are generated in the gas phase using an arc cluster ion source [10] attached to the photoemission electron microscope (PEEM) and are deposited in ultrahigh vacuum under nondestructive (soft landing) conditions onto a 13-nm-thick polycrystalline Co film grown by electron beam evaporation on a silicon substrate (base pressure $5 \times 10^{-10}$ mbar). In order to enable a size-dependent study of the magnetic properties under the same conditions, a wide range of particle sizes is deposited on a single sample. The particles are single crystal with a bcc structure and a magnetization value close to the bulk $[9,10]$. They have a nearly spherical shape (Wulff construction) with only a slight flattening occurring due to the impact on the substrate [11]. Interparticle interactions (dipolar or exchange) can be neglected because of their large nearest-neighbor separation (typically $>500 \mathrm{~nm}$ ), which is also an important prerequisite for PEEM measurements of individual nanoparticles [12,13]. 

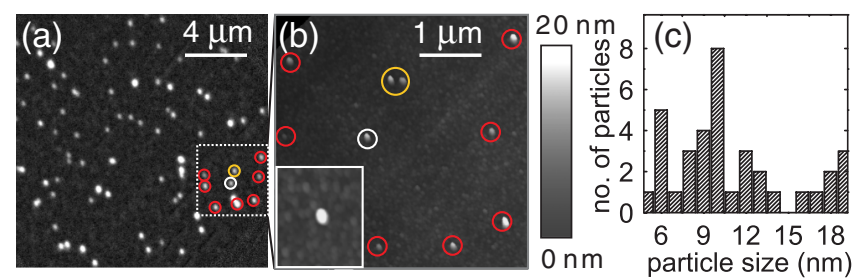

FIG. 1 (color online). (a) PEEM elemental contrast recorded at the $\mathrm{Fe} L_{3}$ edge $(708.1 \mathrm{eV})$ of $\mathrm{Fe}$ nanoparticles with sizes from 5 to $25 \mathrm{~nm}$. (b) AFM image of the marked box in (a) with the dark gray (red) circles showing single nanoparticles and the light gray (yellow) circle indicating two close-lying particles. The inset shows an AFM image of the Fe nanoparticle in the white circle in (a) and (b) with a height of $13 \pm 1 \mathrm{~nm}$. (c) Size distribution of the single Fe nanoparticles in (a).

The magnetic characterization was performed in situ at room temperature using the PEEM at the Surface/ Interface:Microscopy (SIM) beam line of the Swiss Light Source. No traces of oxidation have been detected either in the Fe particles or in the Co film during the PEEM study.

Elemental contrast PEEM images of the Fe nanoparticles [Fig. 1(a)] are obtained by dividing images successively recorded at the $\mathrm{Fe} L_{3}$ absorption edge and at the preedge region. The bright spots correspond to individual Fe particles, and their variation in intensity is mostly related to the varying particle sizes. Since the PEEM spatial resolution is limited to $50 \mathrm{~nm}$, the particle sizes $D$ are determined by subsequent ex situ measurements of the height with an atomic force microscope (AFM). Using chromium-gold lithographic markers on the samples, the same particles are identified with PEEM [Fig. 1(a)] and AFM [Fig. 1(b)]. Close-lying particles [e.g., light gray (yellow) circle in Fig. 1(b)] are excluded from the analysis. An unambiguous identification of single particles was carried out by additional measurements with a scanning electron microscope (not shown). The distribution of particle sizes, which are corrected for the presence of a native surface oxide shell of $2.0 \pm 0.5 \mathrm{~nm}$, is given in Fig. 1(c).

In order to determine the local magnetization orientation of the Fe nanoparticles and the Co film separately, the photon energy was tuned to the $\mathrm{Fe}$ and $\mathrm{Co} L_{3}$ edges (708.1 and $778.1 \mathrm{eV}$ ), respectively. X-ray magnetic circular dichroism (XMCD) contrast images of the Fe nanoparticles [Fig. 2(a)] and of the Co film [Fig. 2(b)] are obtained from the intensity asymmetry ratio of two images sequentially recorded with right- and left-handed circular polarization, $\left(I^{+}-I^{-}\right) /\left(I^{+}+I^{-}\right)$. The XMCD images reflect the projection of the local magnetization $\mathbf{M}$ on the photon propagation vector $\boldsymbol{\sigma}$ and, thus, dissimilar gray levels correspond to different magnetic orientations. The superposition of the $\mathrm{Fe}$ and $\mathrm{Co} \mathrm{XMCD}$ images measured in the same area [Fig. 2(c)] reveals that the magnetization of a large number of $\mathrm{Fe}$ particles is not collinearly aligned to that of the Co substrate. Instead, the images indicate a rich distribution of orientations of the magnetization of the $\mathrm{Fe}$ particles compared to that of the underlying Co domains.

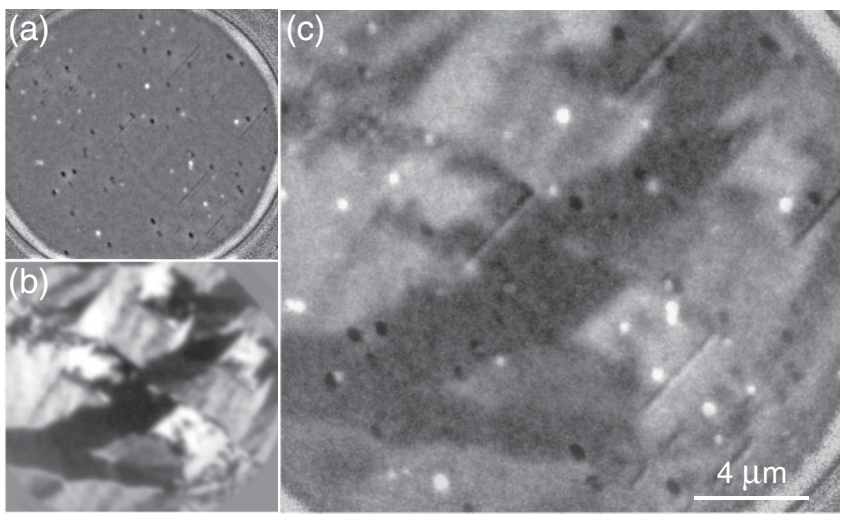

FIG. 2. XMCD magnetic contrast of (a) the Fe nanoparticles shown in Fig. 1(a), and (b) the Co film measured in the same area. (c) Superposition of the Fe and Co XMCD images in (a) and (b). Different gray levels correspond to dissimilar magnetization orientations.

Note that the magnetization of the particles is thermally stable during the whole measurement (several hours).

A quantitative assessment of the magnetization orientation in 3D space of the individual Fe nanoparticles is carried out by recording a series of Fe $L_{3}$-edge XMCD images at different azimuthal sample orientations $\left(\phi_{s}\right)$ relative to $\boldsymbol{\sigma}$ [Fig. 3(a)]. A fit to the angular dependent $\mathrm{XMCD}$ asymmetry ratios, which includes the X-ray incidence angle of $16^{\circ}$, reveals the local magnetization direction in terms of the azimuthal $\phi_{m}$ and polar $\theta_{m}$ angles [Fig. 3(b)], as shown for one selected $\mathrm{Fe}$ particle in Fig. 3(c). The orientation of the magnetization in the Co domains is determined in an analogous manner.

The combination of the XMCD analysis with the particle heights determined from the AFM images reveals a remarkable size dependence of the distribution of the inplane canting angles between the film and the particle magnetization, $\Delta \phi=\phi_{m}(\mathrm{Co})-\phi_{m}(\mathrm{Fe})$, as shown in Fig. 4(a) (white dots). In this figure, we show only nanoparticles which are positioned on regions of well-defined, homogeneous Co magnetization [14]. We find that, while for particles larger than $6 \mathrm{~nm}$ a broad distribution of canting angles is observed even within a narrow size range [see, for example, subset of data around $10 \mathrm{~nm}$ in Fig. 4(a)], the magnetization of particles $\leq 6 \mathrm{~nm}$ is practically collinearly aligned to that of the Co substrate [inset of Fig. 4(a)]. The collinear alignment of the smaller particles, $\Delta \phi=0$, reflects an exchange-dominated regime. For the larger particles, the large variation of canting angles suggests an anisotropy-dominated regime with a wide distribution of easy axes resulting from the random nature of the deposition process.

In order to shed light on these observations, we have evaluated the magnetic energy contributions by performing numerical calculations for particles exchange coupled to a ferromagnetic support. For supported nanoparticles it can be assumed that the contact of the particle with the substrate induces a uniaxial anisotropy $[11,15]$ with an arbi- 


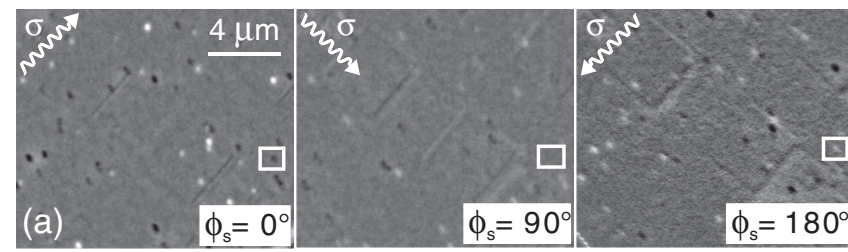

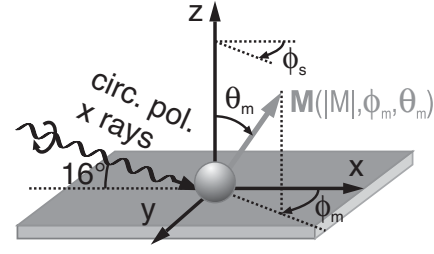

(b)

(c)

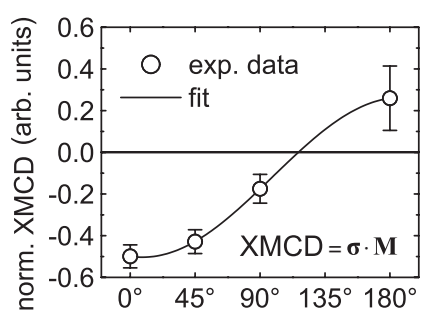

azimuthal orientation $\phi_{S}$
FIG. 3. (a) XMCD images at the Fe $L_{3}$ edge $(708.1 \mathrm{eV})$ of the same region as in Figs. 1(a) and 2 acquired with different azimuthal sample orientations $\phi_{s}$ relative to the photon propagation vector $\boldsymbol{\sigma}$ (indicated by arrows). (b) Sketch of the sample geometry. (c) Quantitative determination of the magnetization direction for the single Fe particle in the inset of Fig. 1(b) [indicated by square frames in Fig. 3(a)]. The fit of the angular dependent XMCD asymmetry ratio gives $\phi_{m}=-170 \pm 30^{\circ}$ and $\theta_{m}=140 \pm 30^{\circ}$.

trary orientation due to the random deposition process. We use a model based on the theoretical framework described by Bruno [16] to calculate the energetically favored spin structure of the nanoparticles in the present size range. For our calculations, we set the easy axis of the particles (arrow labeled $n_{\mathrm{MAE}}$ ) perpendicular to the substrate magnetization $\left(m_{s}\right)$ and parallel to the surface plane. The spins at the particle-substrate interface are kept fixed parallel to $m_{s}$ to take into account the ferromagnetic coupling at the interface, while all of the spins above are free to rotate with an angle $\phi(z)$. The optimal spin structure is determined by minimizing the total energy (integrated over the particle volume) given by the MAE, $E_{\text {ani }}=K_{u} \cos ^{2} \phi$, and the exchange energy, by solving the corresponding Euler equation [16], using the Fe exchange stiffness, $A=62 \mathrm{meV} / \mathrm{m}$ [2]. We find that, for a sufficiently large uniaxial magnetic anisotropy constant $K_{u}$, the optimal arrangement of spins gives rise to a spin spiral, as sketched in Fig. 4(b) (the spin structure corresponds to a particle with $D=10 \mathrm{~nm}$ and $K_{u}=50 \mu \mathrm{eV} /$ atom). Below a certain value of $K_{u}$, the spins are collinearly aligned to $m_{s}$.

Our calculations show in all noncollinear cases that the steepest change in spin orientation occurs close to the interface with the ferromagnetic substrate, while in the upper part of the particle there is an almost parallel arrangement of spins that are oriented at angle $\Delta \phi$ with respect to $m_{s}$. For the particle in Fig. 4(b), the magnetization direction of the upper spins is found to be not at $\Delta \phi=$ $\pm 90^{\circ}$, which would correspond to the easy axis, but at $\Delta \phi= \pm 75^{\circ}$. It is precisely the orientation of these upper spins that is measured in the PEEM due to the finite escape depth of the excited secondary electrons $(2-3 \mathrm{~nm})$ [17].
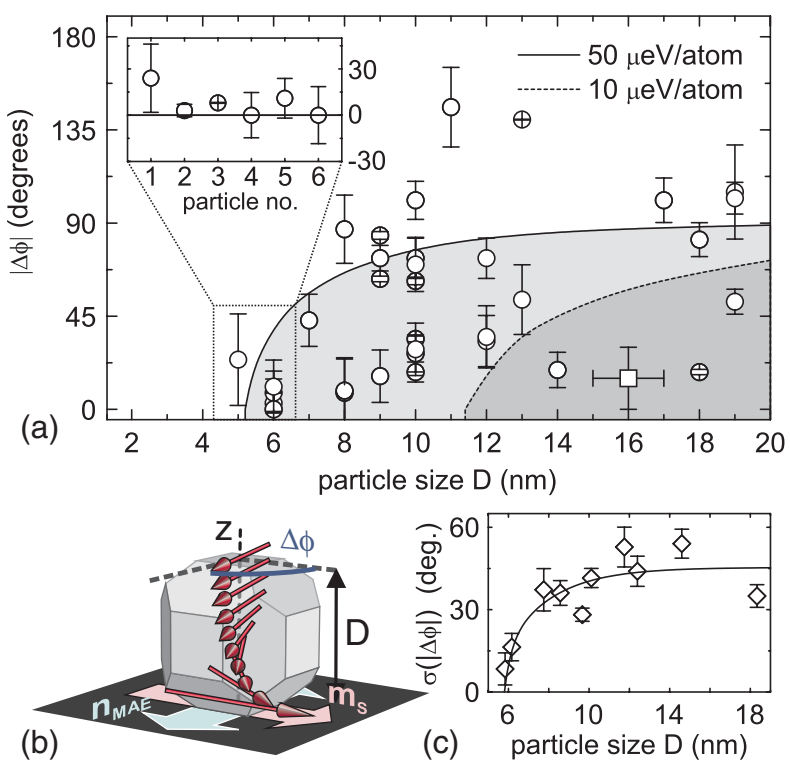

FIG. 4 (color online). (a) Size dependence of the absolute value of the in-plane canting angles between the film and the upper particle magnetization. The white dots are the experimental data with statistical error bars and the solid line and dashed line are the calculated curves for two values of $K_{u}$. The particle with a square symbol shows a representative error bar of the particle size. (b) Sketch of the spin structure of a Wulff-shaped 10-nm particle and $K_{u}=50 \mu \mathrm{eV} /$ atom. (c) Size dependence of the experimental spread of $|\Delta \phi|$ in (a), represented by the standard deviation (diamonds). The solid line is a guide to the eye.

Hence our experiments do not measure an average spin direction of the particle, but correlate directly with the optimal orientation of the uppermost spins. Note that the $\Delta \phi$ calculated with $n_{\mathrm{MAE}}$ perpendicular to $m_{s}$ represents an upper limit for the canting angle. For other orientations of $n_{\mathrm{MAE}}$ the canting angle of the spin spiral is smaller, and tends to zero if $n_{\mathrm{MAE}}$ is parallel to $m_{s}$.

The fact that we have uncovered a spin spiral is remarkable, but another important feature of our model is that it exhibits the kind of size-dependent transition from an exchange-dominated to an anisotropy-dominated regime that we observe in our experiments. The calculated size dependence of the maximum canting angle $\Delta \phi$ is shown by the lines in Fig. 4, corresponding to $K_{u}$ of 10 and $50 \mu \mathrm{eV} /$ atom [values that are close to and larger than that of the cubic bulk Fe $(3.3 \mu \mathrm{eV} /$ atom $)$ [18], respectively]. We find that for $K_{u}=10 \mu \mathrm{eV} /$ atom a canted magnetization is only favored in the model for particles larger than a critical size, $D_{c}$, of $11 \mathrm{~nm}$, while in our experimental data, the transition from collinear to noncollinear states is found at $6 \mathrm{~nm}$ [Fig. 4(a)]. This already shows that the particles must have a $K_{u}$ larger than $10 \mu \mathrm{eV} /$ atom. For $K_{u}=50 \mu \mathrm{eV} /$ atom the formation of a spin spiral becomes favorable only for particles larger than $D_{c}=5 \mathrm{~nm}$, while for smaller particles a collinear alignment with $m_{s}$ is preferred. Above $5 \mathrm{~nm}$, the deviation from the collinear alignment to the substrate magnetization 
increases until eventually the particle's easy axis direction, $\Delta \phi= \pm 90^{\circ}$ in the calculations, is reached at a size of $20 \mathrm{~nm}$. Thus the model reproduces qualitatively the experimental trend [white dots in Fig. 4(a)]: $\Delta \phi$ close to zero for particles $\leq 6 \mathrm{~nm}$ and a broad distribution of $\Delta \phi$ for larger particles. This is better observed in Fig. 4(c) where the spread of $|\Delta \phi|$ shows a clear transition from collinear to noncollinear alignment at a critical size of $\sim 6 \mathrm{~nm}$. A further analysis of our calculations reveals an empirical relationship between the Bloch-wall width, $w=2 \sqrt{A / K_{u}}$, and the critical size for the spin-spiral formation, $D_{c}=$ $2 / 3 w$, below which the exchange coupling is dominant. This relationship allows one to predict the critical size for spin-spiral formation of nanoparticles if the actual values of $A$ and $K_{u}$ are known.

A more quantitative comparison between the model and the experiments is also possible, and permits an estimation of a lower limit for the anisotropy constant of the particles. The fact that the actual direction of the particle anisotropy axis is unknown precludes a direct fit to the curves of maximum canting angle. Nevertheless, we can establish the lower threshold of $K_{u}$ of the particles required to reproduce the experimental observations. We see that the canting angle of the majority of the particles is larger than the calculated curve of $K_{u}=10 \mu \mathrm{eV} /$ atom (dashed line) and that the curve for $K_{u}=50 \mu \mathrm{eV} /$ atom (solid line) provides an approximate envelope for our data. This shows that there appears to be a significant modification of the anisotropy of the Fe particles compared to that of the cubic Fe bulk. Two possible mechanisms for this surprising enhancement of $K_{u}$ are interface-induced strain due to particle-substrate contact forces and partial alloying at the interface, which occurs upon impact of the particles on the substrate. The first mechanism is supported both by first-principles density-functional calculations, which give anisotropy values of up to $100 \mu \mathrm{eV} /$ atom for even moderate tetragonal lattice distortions $(c / a \sim 1.05)$ [19], and by coherent $x$-ray diffraction experiments, which reveal complex strain patterns in supported nanoparticles [20]. Regarding the second mechanism, anisotropies of up to $1 \mathrm{meV} /$ atom are found for tetragonally distorted $\mathrm{FeCo}$ alloys [21]. The partial flattening of the particles upon deposition is estimated to be less important [11]. It should be noted that our calculations neglect the effect of dipolar interactions and surface variations of the spin structure (e.g., curling) which might be addressed by more sophisticated approaches $[22,23]$. This is justified since the dipolar contribution is small compared to the exchange and anisotropy energies and the curling will only have a minor effect on the spin spiral.
In conclusion, direct observations of the magnetization orientation of individual $\mathrm{Fe}$ nanoparticles on a ferromagnetic Co film show a transition from an exchangedominated to an anisotropy-dominated regime at a size of $\sim 6 \mathrm{~nm}$. Numerical calculations reveal a spin-spiral structure in the particles above the critical size. This demonstrates that unexpected spin arrangements at nanoscale interfaces can result from the balance between the particle-substrate interaction and the individual properties of the particles. This knowledge is crucial for the development of novel devices which make use of nanoscale magnetic phenomena at interfaces.

We thank K.-H. Meiwes-Broer (University of Rostock), A. Weber and R. Schelldorfer (Paul Scherrer Institut), and funding by the DFG-SPP 1153 [Grants No. BA 1612/3-3 and No. KL 2188/1-3] and the EC FP7 [Grant No. NMP3SL-2008-214469]. Part of this work was performed at the Swiss Light Source, Paul Scherrer Institut, Villigen, Switzerland.

*arantxa.fraile-rodriguez@psi.ch

[1] J. Bansmann et al., Surf. Sci. Rep. 56, 189 (2005).

[2] R. C. O'Handley, Modern Magnetic Materials: Principles and Applications (Wiley-VCH, Weinheim, 2000).

[3] A. Yamasaki et al., Phys. Rev. Lett. 91, 127201 (2003).

[4] S. Krause et al., Phys. Rev. Lett. 103, 127202 (2009).

[5] E. E. Fullerton, J.S. Jiang, and S.D. Bader, J. Magn. Magn. Mater. 200, 392 (1999).

[6] V. Skumryev et al., Nature (London) 423, 850 (2003).

[7] J. T. Lau et al., Phys. Rev. Lett. 89, 057201 (2002).

[8] K. Edmonds et al., J. Magn. Magn. Mater. 231, 113 (2001).

[9] A. Kleibert, K.-H. Meiwes-Broer, and J. Bansmann, Phys. Rev. B 79, 125423 (2009).

[10] A. Kleibert et al., J. Appl. Phys. 101, 114318 (2007).

[11] A. Kleibert et al., J. Phys. Condens. Matter 20, 445005 (2008).

[12] J. Rockenberger et al., J. Chem. Phys. 116, 6322 (2002).

[13] A. Fraile Rodríguez et al., J. Magn. Magn. Mater. 316, 426 (2007).

[14] L. J. Heyderman et al., J. Appl. Phys. 99, 063904 (2006).

[15] M. Jamet et al., Phys. Rev. B 69, 024401 (2004).

[16] P. Bruno, Phys. Rev. Lett. 83, 2425 (1999).

[17] H. Ohldag et al., Phys. Rev. Lett. 87, 247201 (2001).

[18] H. P. Klein and E. Kneller, Phys. Rev. 144, 372 (1966).

[19] T. Burkert et al., Phys. Rev. B 69, 104426 (2004).

[20] M. A. Pfeifer et al., Nature (London) 442, 63 (2006).

[21] G. Andersson et al., Phys. Rev. Lett. 96, 037205 (2006).

[22] R. Hertel et al., Phys. Rev. B 72, 214409 (2005).

[23] N. Kazantseva et al., Phys. Rev. B 77, 184428 (2008). 